side effects were significantly greater with dextroamphetamine, particularly insomnia, irritability, crying, anxiousness, dysphoria, and nightmares. Dextroamphetamine is the preferred first-line stimulant for ADHD in Australia because of cost constraints. However, this study demonstrates the superiority of methylphenidate over an amphetamine, and in the US, MPH should be the drug of choice. Dextroamphetamine and Adderal are alternative therapies of proven value.

Short- and long-term safety and efficacy of stimulants in ADHD is reviewed by Greenhill LL et al. (I Am Acad Child Adolesc Psychiatry May 1999;38:503-512). Long-term use of stimulants is not considered harmful.

\title{
NEUROCOGNITIVE DEFICITS IN ADOLESCENTS BORN PRETERM
}

Neurocognitive and behavioral outcome at adolescence of 105 infants born very preterm ( $<33$ weeks) was studied and correlated with neonatal ultrasonographic scans and MRI at age 14-15 years, at University College London Medical School, UK. Of 72 cases completing the study, 40 had abnormal and 15 equivocal MRIs, markers of hypoxic-ischemic damage, especially ventricular dilatation, atrophy of the corpus callosum - predominantly posterior, and white matter abnormalities. In contrast, of 21 controls, one had abnormal and 5 equivocal MRI. MRI detected more abnormalities than neonatal ultrasonography (40 of 72 vs 31 of 72). Tests of functional neurodevelopment and behavior at age 14-15 years showed significantly more reading, adjustment, and neurological impairments in the preterm cases than controls, but only the impaired behavior on the Rutter scale was significantly related to structural brain abnormalities on MRI. Four cases and no controls had epilepsy, and all four had abnormal MRI scans. (Stewart AL, Rifkin L, Amess PN et al. Brain structure and neurocognitive and behavioral function in adolescents who were born very preterm. Lancet May 15 1999;353:1653-57). (Respond: Dr AL Stewart, Perinatal Brain Research Group, Department of Paediatrics, Rayne Institute, University College London Medical School, London WC1E 6JJ, UK).

COMMENT. Very preterm infants are at high risk of neurocognitive and behavioral problems and MRI abnormalities in adolescence, and abnormalities in the MRI, notably in the corpus callosum, are significantly correlated with neurobehavioral disorders. MRI identifies more brain lesions than does neonatal ultrasonography.

Callosal and cortical contribution to procedural learning has been studied in eleven adult patients, 6 acallosal and 5 callosotomized, at the University of Montreal, Canada. (de Guise E, del Pesce M, Foschi N et al. Brain June 1999;122:1049-1062). Epileptic foci in frontal or temporal areas occurred in 7 and post-surgical bilateral prefrontal atrophy in one. Performance of a serial reaction time task, involving bimanual or unimanual key-pressing responses to a sequence of repeated visual stimuli, showed that the anterior part of the corpus callosum is crucial for integration and transfer of a procedural visuomotor skill, and the frontal lobes are important for unilateral procedural learning. Acallosal subjects have explicit knowledge of the procedure but are incapable of learning the skill implicitly when interhemispheric integration is required. In agenesis of the corpus callosum subjects, there is a disconnection deficit and a dissociation of declarative (explicit) and procedural (implicit) memory.

Visuomotor learning, a procedural memory task, refers to a motor skill or cognitive routine acquired through practice. This type of implicit memory, 
requiring the integrity of the frontal lobes and corpus callosum, is expressed by a reduction of reaction time or errors over trials. In contrast, declarative memory, mediated by a corticothalamocortical circuit, is the ability to store and recall visual pictures, words or events. The anatomy of memory is discussed in Progress in Pediatric Neurology II, PNB Publ, 1994;pp178-9.

\section{SEIZURE DISORDERS}

\section{OUTCOME OF LATE FEBRILE SEIZURES}

The clinical and EEG features, the occurrence of subsequent seizures, and the neurologic outcome in 50 children who had febrile seizures (FS) after 5 years of age were studied at the University of British Columbia, Canada. FS had occurred before 5 years of age in 38 , and recurred before 5 years in 27 . In addition to this group with both early and late FS occurrence, 12 had the first FS after 5 years of age. There was no significant difference between these two groups with respect to early history, EEG or outcome. During a 1-13 year follow-up (median, 5 yrs 6 mths), 40 had two or less FS after 5 years, and none occurred after 10 years of age. FS were complex in $20(40 \%)$, and $16(32 \%)$ had a family history of FS in a firstdegree relative. Afebrile seizures developed in 5 (10\%), 18 (36\%) had school difficulties, and 9 (18\%) had abnormal psychological tests. Learning difficulties in 18 were associated with developmental delay in $33 \%$, motor incoordination in 44\%, and afebrile seizures in 22\%. Epileptiform EEGs occurred in 22 (44\%). (Webb DW, Jones RR, Manzur AY, Farrell K. Retrospective study of late febrile seizures. Pediatr Neurol April 1999;20:270-273). (Respond: Dr Kevin Farrell, Department of Paediatrics, University of British Columbia, 4480 Oak Street, Vancouver, British Columbia, V6H 3V4, Canada).

COMMENT. Children with febrile seizures that occur late, after 5 years of age, have a higher frequency of recurrence of early onset FS, complex FS in $40 \%$, a remission of FS before 10 years, a strong family history of FS, afebrile seizures in $10 \%$, and a risk of learning disabilities in one third. Other factors known to influence the outcome of FS, such as the height of the fever at the time of the seizure ("FS threshold"), and the duration of the FS, would have been of interest (Millichap JG. Febrile Convulsions, New York, Macmillan, 1968). The 32\% familial incidence observed in the Canadian study is similar to that in an unselected group of 95 FS patients followed in my own clinic. The evidence for an inherited predisposition to FS was equally strong in those who had afebrile in addition to FS.

Rectal diazepam gel for treatment of acute repetitive seizures in children at home is evaluated by an analysis of pediatric data from two previously published reports of the effects of Diastat (DZP) in children and adults (Kriel RL, Cloyd JC, Pellock JM et al. Pediatr Neurol April 1999;20:282-288). Comparing the results of 68 DZP-treated children and 65 receiving placebo, the DZP group showed a significant reduction in median seizure frequency, and more DZP-treated children remained seizure free at the completion of the 12-hour observation period (40 vs 20, $\mathrm{P}=.001$ ). Doses of Diastat were based on age and weight: $0.5 \mathrm{mg} / \mathrm{kg}$ for ages 2-5 years; $0.3 \mathrm{mg} / \mathrm{kg}$ for ages $6-11$ years; and $0.2 \mathrm{mg} / \mathrm{kg}$ at 12 years and older. Somnolence occurred more frequently in the Diastat group of patients (25\% vs $7.7 \%, \mathrm{P}=.009$ ), but no instance of serious respiratory depression was reported. Rectal DZP is effective and relatively safe when used to abort an episode of acute repetitive seizures in a child, and seizure recurrence is lessened in a 12 hour observation period. A single dose is recommended, but if seizures are refractory, a 